\title{
Factorization of Block Triangular Matrix Functions with Off-diagonal Binomials
}

\author{
Cornelis V.M. van der Mee, Leiba Rodman and Ilya M. Spitkovsky
}

Dedicated to Israel Gohberg on the occasion of his 75th birthday

\begin{abstract}
Factorizations of Wiener-Hopf type are considered in the abstract framework of Wiener algebras of matrix-valued functions on connected compact abelian groups, with a non-archimedean linear order on the dual group. A criterion for factorizability is established for $2 \times 2$ block triangular matrix functions with elementary functions on the main diagonal and a binomial expression in the off-diagonal block.
\end{abstract}

Mathematics Subject Classification (2000). Primary 47A68. Secondary 43A17.

Keywords. Wiener-Hopf factorization, Wiener algebras, linearly ordered groups.

\section{Introduction and the main result}

Let $G$ be a (multiplicative) connected compact abelian group and let $\Gamma$ be its (additive) character group. Recall that $\Gamma$ consists of continuous homomorphisms of $G$ into the group of unimodular complex numbers. Since $G$ is compact, $\Gamma$ is discrete. In applications, often $\Gamma$ is an additive subgroup of $\mathbb{R}$, the group of real numbers, or of $\mathbb{R}^{k}$, and $G$ is the Bohr compactification of $\Gamma$. The group $G$ can be also thought of as the character group of $\Gamma$, an observation that will be often used.

The group $G$ has a unique invariant measure $\nu$ satisfying $\nu(G)=1$, while $\Gamma$ is equipped with the discrete topology and the (translation invariant) counting measure. It is well known [17] that, because $G$ is connected, $\Gamma$ can be made into a linearly ordered group. So let $\preceq$ be a linear order such that $(\Gamma, \preceq)$ is an ordered group, i. e., if $x, y, z \in \Gamma$ and $x \preceq y$, then $x+z \preceq y+z$. Throughout the paper it will

The research of van der Mee leading to this article was supported by INdAM and MIUR under grants No. 2002014121 and 2004015437. The research of Rodman and Spitkovsky was partially supported by NSF grant DMS-9988579. 
be assumed that $\Gamma$ is ordered with a fixed linear order $\preceq$. The notations $\prec, \succeq, \succ$, max, min (with obvious meaning) will also be used. We put $\Gamma_{+}=\{x \in \Gamma: x \succeq 0\}$ and $\Gamma_{-}=\{x \in \Gamma: x \preceq 0\}$.

For any nonempty set $M$, let $\ell^{1}(M)$ stand for the complex Banach space of all complex-valued $M$-indexed sequences $\boldsymbol{x}=\left\{x_{j}\right\}_{j \in M}$ having at most countably many nonzero terms that are finite with respect to the norm

$$
\|\boldsymbol{x}\|_{1}=\sum_{j \in M}\left|x_{j}\right|
$$

Then $\ell^{1}(\Gamma)$ is a commutative Banach algebra with unit element with respect to the convolution product $(\boldsymbol{x} * \boldsymbol{y})_{j}=\sum_{k \in \Gamma} x_{k} y_{j-k}$. Further, $\ell^{1}\left(\Gamma_{+}\right)$and $\ell^{1}\left(\Gamma_{-}\right)$are closed subalgebras of $\ell^{1}(\Gamma)$ containing the unit element.

Given $a=\left\{a_{j}\right\}_{j \in \Gamma} \in \ell^{1}(\Gamma)$, by the symbol of $a$ we mean the complex-valued continuous function $\hat{a}$ on $G$ defined by

$$
\hat{a}(g)=\sum_{j \in \Gamma} a_{j}\langle j, g\rangle, \quad g \in G,
$$

where $\langle j, g\rangle$ stands for the action of the character $j \in \Gamma$ on the group element $g \in G$ (thus, $\langle j, g\rangle$ is a unimodular complex number), or, by Pontryagin duality, of the character $g \in G$ on the group element $j \in \Gamma$. The set

$$
\sigma(\hat{a}):=\left\{j \in \Gamma: a_{j} \neq 0\right\}
$$

will be called the Fourier spectrum of $\hat{a}$ given by (1). Since $\Gamma$ is written additively and $G$ multiplicatively, we have

$$
\begin{gathered}
\langle\alpha+\beta, g\rangle=\langle\alpha, g\rangle \cdot\langle\beta, g\rangle, \quad \alpha, \beta \in \Gamma, \quad g \in G, \\
\langle\alpha, g h\rangle=\langle\alpha, g\rangle \cdot\langle\alpha, h\rangle, \quad \alpha \in \Gamma, \quad g, h \in G .
\end{gathered}
$$

We will use the shorthand notation $e_{\alpha}$ for the function $e_{\alpha}(g)=\langle\alpha, g\rangle, g \in G$. Thus, $e_{\alpha+\beta}=e_{\alpha} e_{\beta}, \alpha, \beta \in \Gamma$.

The set of all symbols of elements $a \in \ell^{1}(\Gamma)$ forms an algebra $W(G)$ of continuous functions on $G$. The algebra $W(G)$ (with pointwise multiplication and addition) is isomorphic to $\ell^{1}(\Gamma)$. Denote by $W(G)_{+}$(resp., $W(G)_{-}$) the algebra of symbols of elements in $\ell^{1}\left(\Gamma_{+}\right)$(resp., $\left.\ell^{1}\left(\Gamma_{-}\right)\right)$.

We have the following result. For every unital Banach algebra $\mathcal{A}$ we denote its group of invertible elements by $\mathcal{G}(\mathcal{A})$.

Theorem 1. Let $G$ be a compact abelian group with character group $\Gamma$, and let $W(G)^{n \times n}$ be the corresponding Wiener algebra of $n \times n$ matrix functions. Then $\hat{A} \in \mathcal{G}\left(W(G)^{n \times n}\right)$ if and only if $\hat{A}(g) \in \mathcal{G}\left(\mathbb{C}^{n \times n}\right)$ for every $g \in G$.

This is an immediate consequence of Theorem A.1 in [8] (also proved in [1], and see [14]).

We now consider the discrete abelian subgroup $\Gamma^{\prime}$ of $\Gamma$ and denote its character group by $G^{\prime}$. Then we introduce the annihilator

$$
\Lambda=\left\{g \in G:\langle j, g\rangle=1 \text { for all } j \in \Gamma^{\prime}\right\},
$$


which is a closed subgroup of $G$ and hence a compact group. According to Theorem 2.1.2 in [17], we have $G^{\prime} \simeq(G / \Lambda)$.

Let us now introduce the natural projection $\pi: G \rightarrow G / \Lambda$. We observe that the above theorem also applies to $W\left(G^{\prime}\right)^{n \times n}$. Given $A \in \ell^{1}(\Gamma)^{n \times n}$ with its Fourier spectrum restricted to $\Gamma^{\prime}$ (i.e., $A_{j}=0$ for $j \in \Gamma \backslash \Gamma^{\prime}$ ), we have two symbol definitions:

$$
\begin{aligned}
& \hat{A}_{\Gamma}(g)=\sum_{j \in \Gamma^{\prime}} A_{j}\langle j, g\rangle, \quad g \in G, \\
& \hat{A}_{\Gamma^{\prime}}(g)=\sum_{j \in \Gamma^{\prime}} A_{j}\langle j, g\rangle, \quad g \in G^{\prime},
\end{aligned}
$$

where we have taken into account that $A_{j}=0$ for $j \in \Gamma \backslash \Gamma^{\prime}$. The latter can be replaced by

$$
\hat{A}_{\Gamma^{\prime}}([g])=\sum_{j \in \Gamma^{\prime}} A_{j}\langle j, g\rangle, \quad[g] \in(G / \Lambda),
$$

where $[g]=\pi(g)$ for $g \in G$. Obviously, $\langle j, g\rangle$ only depends on $[g]=\pi(g)$ if $j \in \Gamma^{\prime}$. (If $\left[g_{1}\right]=\left[g_{2}\right]$, then $g_{1} g_{2}^{-1} \in \Lambda$ and hence $\left\langle j, g_{1} g_{2}^{-1}\right\rangle=1$ for all $j \in \Gamma^{\prime}$, which implies the statement.) Thus the two symbol definitions are equivalent in the sense that the value of "the" symbol $\hat{A}$ on $g \in G$ only depends on $[g]=\pi(g)$.

Theorem 2. Let $\Gamma^{\prime}$ be a subgroup of the discrete abelian group $\Gamma$, let $G$ and $G^{\prime}$ be the character groups of $\Gamma$ and $\Gamma^{\prime}$, respectively, and let $\Lambda$ be defined by (2). If $\hat{A} \in W(G)^{n \times n}$ is an element which has all of its Fourier spectrum within $\Gamma^{\prime}$, then $\hat{A} \in \mathcal{G}\left(W\left(G^{\prime}\right)^{n \times n}\right)$ if and only if $\hat{A}(g) \in \mathcal{G}\left(\mathbb{C}^{n \times n}\right)$ for every $g \in G$.

For the proof see [14].

We now consider factorizations. A (left) factorization of $A \in(W(G))^{n \times n}$ is a representation of the form

$$
A(g)=A_{+}(g)\left(\operatorname{diag}\left(e_{j_{1}}(g), \ldots, e_{j_{n}}(g)\right)\right) A_{-}(g), \quad g \in G,
$$

where $A_{+} \in \mathcal{G}\left(\left(W(G)_{+}\right)^{n \times n}\right), A_{-} \in \mathcal{G}\left(\left(W(G)_{-}\right)^{n \times n}\right)$, and $j_{1}, \ldots, j_{n} \in \Gamma$. Here and elsewhere we use $\operatorname{diag}\left(x_{1}, \ldots, x_{n}\right)$ to denote the $n \times n$ diagonal matrix with $x_{1}, \ldots, x_{n}$ on the main diagonal, in that order. The elements $j_{k}$ are uniquely defined (if ordered $j_{1} \preceq j_{2} \preceq \cdots \preceq j_{n}$ ); this can be proved by a standard argument (see [9, Theorem VIII.1.1]). The elements $j_{1}, \ldots, j_{n}$ in (3) are called the (left) factorization indices of $A$.

If all factorization indices coincide with the zero element of $\Gamma$, the factorization is called canonical. If a factorization of $A$ exists, the function $A$ is called factorizable. For $\Gamma=\mathbb{Z}$ and $G$ the unit circle, the definitions and the results are classical [10], [9], [4]; many results have been generalized to $\Gamma=\mathbb{R}^{k}$ (see [2] and references there), and $\Gamma$ a subgroup of $\mathbb{R}^{k}$ (see $[15],[16]$ ). The notion of factorization in the abstract abelian group setting was introduced and studied, in particular, for block triangular matrices, in [14]. The present paper can be thought of as a follow up of [14]. 
In this paper we prove the following result.

Theorem 3. Let $A$ have the form

$$
A(g)=\left[\begin{array}{cc}
e_{\lambda}(g) I_{p} & 0 \\
c_{1} e_{\sigma}(g)-c_{2} e_{\mu}(g) & e_{-\lambda}(g) I_{q}
\end{array}\right], \quad g \in G,
$$

and assume that $\lambda \succ 0, \mu \succ \sigma$, and

$$
n \mu \prec \lambda, \quad n \sigma \prec \lambda \text { for all integers } n \text {. }
$$

Then $A$ admits a factorization if and only if

$$
\begin{aligned}
\operatorname{rank}\left(\lambda_{1} c_{1}-\lambda_{2} c_{2}\right)= & \max \left\{\operatorname{rank}\left(z_{1} c_{1}-z_{2} c_{2}\right): z_{1}, z_{2} \in \mathbb{C}\right\} \\
& \text { for every } \lambda_{1}, \lambda_{2} \in \mathbb{C} \text { satisfying }\left|\lambda_{1}\right|=\left|\lambda_{2}\right|=1 .
\end{aligned}
$$

Moreover, in case a factorization exists, the factorization indices of $A$ belong to the set

$$
\{ \pm \sigma, \pm \mu, \pm \lambda, \lambda-(\mu-\sigma), \ldots, \lambda-\min \{p, q\}(\mu-\sigma)\} .
$$

We emphasize that the setting of Theorem 3 is a non-archimedean linearly ordered abelian group $(\Gamma, \succeq)$, in contrast with the archimedean linear order of $\mathbb{R}$ and its subgroups. The setting of non-archimedean, as well as archimedean, linearly ordered abelian subgroups was studied in [14].

\section{Preliminary results on factorization}

Theorem 4. If $A$ admits a factorization (3), and if the Fourier spectrum $\sigma(A)$ is bounded:

$$
\lambda_{\min } \preceq \sigma(A) \preceq \lambda_{\max },
$$

for some $\lambda_{\min }, \lambda_{\max } \in \Gamma$, then the factorization indices are also bounded with the same bounds

$$
\lambda_{\min } \preceq j_{k} \preceq \lambda_{\max }, \quad k=1,2, \ldots, n,
$$

and moreover,

$$
\sigma\left(A_{-}\right) \subseteq\left\{j \in \Gamma:-\lambda_{\max }+\lambda_{\min } \preceq j \preceq 0\right\}
$$

and

$$
\sigma\left(A_{+}\right) \subseteq\left\{j \in \Gamma: 0 \preceq j \preceq \lambda_{\max }-\lambda_{\min }\right\} .
$$

Proof. We follow well-known arguments. Rewrite (3) in the form

$$
A_{+}^{-1}\left(e_{-\lambda_{\min }} A\right)=e_{-\lambda_{\min }} \Lambda A_{-} .
$$

Since the left-hand side is in $W(G)_{+}^{n \times n}$, so is the right-hand side, and we have $j_{k} \succeq \lambda_{\min }$ for all $k=1, \ldots, n$ (otherwise, $A_{-}$would contain a zero row, which is impossible because $A_{-}$is invertible). Analogously the second inequality in (7) is proved. Now

$$
e_{\lambda_{\max }-\lambda_{\min }} A_{-}=\left(e_{\lambda_{\max }} \Lambda^{-1}\right) A_{+}^{-1}\left(e_{-\lambda_{\min }} A\right)
$$


is a product of three matrix functions in $W(G)_{+}^{n \times n}$, and therefore also

$$
e_{\lambda_{\max }-\lambda_{\min }} A_{-} \in W(G)_{+}^{n \times n} .
$$

This proves (8); (9) is proved analogously.

It follows from the proof that "one-sided" bounds are valid for the factorization indices:

$$
\begin{array}{lll}
\lambda_{\min } \preceq \sigma(A) & \Longrightarrow & \lambda_{\min } \preceq j_{k}, \text { for } k=1,2, \ldots, n ; \\
\sigma(A) \preceq \lambda_{\max } \quad \Longrightarrow & j_{k} \preceq \lambda_{\max } \text { for } k=1,2, \ldots, n .
\end{array}
$$

For future use we record the next corollary of Theorem 4 . On $\Gamma_{+} \backslash\{0\}$ we consider the equivalence relation (cf. [6])

$$
i \sim j \Longleftrightarrow \exists n, m \in \mathbb{N}:(n i \succ j \text { and } m j \succ i) .
$$

Here $\mathbb{N}$ is the set of positive integers. Any such $i, j$ are called archimedeally equivalent (with respect to $(\Gamma, \preceq)$ ). The $\operatorname{set} \operatorname{Arch}(\Gamma, \preceq)$ of archimedean equivalence classes, which are additive semigroups (in the sense that they are closed under addition), can be linearly ordered in a natural way. Given $\boldsymbol{J} \in \operatorname{Arch}(\Gamma, \preceq)$, it is easily seen that

$$
\Gamma_{\boldsymbol{J}}:=\{i-j: i, j \in \boldsymbol{J}\}
$$

is the smallest additive subgroup of $\Gamma$ containing $\boldsymbol{J}$ and that $\Gamma_{\boldsymbol{J}}$ in fact contains all archimedean components $\preceq \boldsymbol{J}$ in $\operatorname{Arch}(\Gamma, \preceq)$.

Before proceeding we first discuss some illustrative examples.

a. If $\mathbb{Z}^{k}$ is ordered lexicographically, in increasing order the archimedean components are as follows: $J_{0}=(0), J_{1}=(0)_{k-1} \times \mathbb{N}, J_{2}=(0)_{k-2} \times \mathbb{N} \times \mathbb{Z}$, $J_{3}=(0)_{k-3} \times \mathbb{N} \times \mathbb{Z}^{2}, \ldots, J_{k-1}=(0)_{1} \times \mathbb{N} \times \mathbb{Z}^{k-2}$, and $J_{k}=\mathbb{N} \times \mathbb{Z}^{k-1}$.

b. $\mathbb{Z}^{2}$ with linear order $\left(i_{1}, i_{2}\right) \succ(0,0)$ whenever $i_{1}+i_{2} \sqrt{5}>0$. Then the ordered group is archimedean and in increasing order the archimedean components are $J_{0}=(0)$ and $J_{1}=\left\{i \in \mathbb{Z}^{2}: i \succ 0\right\}$.

c. Let $\left(i_{1}, i_{2}\right) \succ(0,0)$ whenever $i_{1}+i_{2}>0$. Then in increasing order the archimedean components are $J_{0}=(0), J_{1}=\{(j,-j): j \in \mathbb{N}\}$, and $J_{2}=$ $\left\{\left(i_{1}, i_{2}\right): i_{1}+i_{2}>0\right\}$.

We now have the following corollary.

Corollary 5. If $A$ admits a factorization (3), and if the Fourier spectrum $\sigma(A)$ is contained in $\Gamma_{\boldsymbol{J}}$ for some $\boldsymbol{J} \in \operatorname{Arch}(\Gamma, \preceq)$, then

$$
j_{k} \in \Gamma_{\boldsymbol{J}}, \quad k=1, \ldots, n,
$$

and

$$
\sigma\left(A_{-}^{ \pm 1}\right) \in \Gamma_{\boldsymbol{J}}, \quad \sigma\left(A_{+}^{ \pm 1}\right) \in \Gamma_{\boldsymbol{J}}
$$

Indeed, in addition to using Theorem 4 we need only to observe that if $X \in$ $\mathcal{G}\left(W(G)^{n \times n}\right)$ is such that $\sigma(X) \subseteq \Gamma^{\prime}$ for some subgroup $\Gamma^{\prime} \subseteq \Gamma$, then $\sigma\left(X^{-1}\right) \subseteq$ $\Gamma^{\prime}$, and apply this observation for $X=A_{ \pm}$(the observation follows easily from Theorem 2). 
Corollary 5 may be considered as asserting the hereditary property of Fourier spectra for additive subgroups of $\Gamma$ of the form $\Gamma_{j}$. We say that a subgroup $\Gamma^{\prime}$ of $\Gamma$ has the hereditary property if for each matrix function $A$ that admits a factorization (3), and the Fourier spectrum of $A$ is contained in $\Gamma^{\prime}$, we have that the factorization indices as well as the Fourier spectra of $A_{ \pm}$and of $A_{ \pm}^{-1}$ are also contained in $\Gamma^{\prime}$. This notion was introduced in [16] for $\Gamma$ the additive group $\mathbb{R}^{k}$; the hereditary property of certain subgroups of $\mathbb{R}^{k}$ was proved there as well. It is an open question whether or not the hereditary property holds for every subgroup of the character group of every connected compact abelian group.

A factorization (3) will be called finitely generated if the Fourier spectra of $A_{+}$and of $A_{-}$are contained in some finitely generated subgroup of $\Gamma$. Clearly, a necessary condition for existence of a finitely generated factorization of $A$ is that the Fourier spectrum of $A$ is contained in a finitely generated subgroup of $\Gamma$. We shall prove below that this condition is also sufficient.

In the proof of the following theorem we make use of a natural projection: If $B \in W(G)^{n \times n}$ is given by the series

$$
B(g)=\sum_{j \in \Gamma} B_{j}\langle j, g\rangle, \quad g \in G,
$$

and if $\Omega$ is a subset of $\Gamma$, we define $B_{\Omega}$ by

$$
B_{\Omega}(g)=\sum_{j \in \Omega} B_{j}\langle j, g\rangle, \quad g \in G .
$$

Clearly, $B_{\Omega} \in W(G)^{n \times n}$ and the Fourier spectrum of $B_{\Omega}$ is contained in $\Omega$.

Theorem 6. If $A \in W(G)^{n \times n}$ is factorizable, and if the Fourier spectrum of $A$ is contained in a finitely generated subgroup of $\Gamma$, then $A$ admits a finitely generated factorization.

Proof. Let $\tilde{\Gamma}$ be a finitely generated subgroup of $\Gamma$ that contains the Fourier spectrum of $A$. Let (3) be a factorization of $A$. Since $\left(W(G)_{ \pm}\right)^{n \times n}$ are unital Banach algebras, the set of invertible elements $\mathcal{G}\left(\left(W(G)_{ \pm}\right)^{n \times n}\right)$ is open in $\left(W(G)_{ \pm}\right)^{n \times n}$. Thus, there exists a finitely generated subgroup $\breve{\Gamma}$ of $\Gamma$ with the following properties:

(a) $\breve{\Gamma}$ contains $\tilde{\Gamma}$;

(b) $\breve{\Gamma}$ contains the elements $j_{1}, \ldots, j_{n}$;

(c) $\left(A_{-}\right)_{\Omega}$ and $\left(A_{+}^{-1}\right)_{\Omega}$ are invertible in $\left(W(G)_{ \pm}\right)^{n \times n}$ for every set $\Omega \supseteq \breve{\Gamma}$.

For verification of (c), note the following estimate:

$$
\begin{aligned}
\left\|A_{-}-\left(A_{-}\right)_{\Omega}\right\|_{\left(W(G)_{ \pm}\right)^{n \times n}} & =\sum_{j \in \Gamma \backslash \Omega}\left\|\left(A_{-}\right)_{j}\right\| \leq \sum_{j \in \Gamma \backslash \breve{\Gamma}}\left\|\left(A_{-}\right)_{j}\right\| \\
& =\left\|A_{-}-\left(A_{-}\right)_{\breve{\Gamma}}\right\|_{\left(W(G)_{ \pm}\right)^{n \times n}} .
\end{aligned}
$$

Letting $\breve{G}$ be the dual group of $\breve{\Gamma}$, by Theorem 2 and (3) we have

$$
\left(A_{+}^{-1}\right)_{\breve{\Gamma}},\left(A_{-}\right)_{\breve{\Gamma}} \in \mathcal{G}\left(\left(W(\breve{G})_{ \pm}\right)^{n \times n}\right) .
$$


Rewrite the equality (3) in the form

$$
\left(A_{+}(g)\right)^{-1} A(g)=\left(\operatorname{diag}\left(e_{j_{1}}(g), \ldots, e_{j_{n}}(g)\right)\right) A_{-}(g) .
$$

Write also (omitting the argument $g \in G$ in the formulas)

$$
\begin{aligned}
& \left(\left(A_{+}^{-1}\right)_{\breve{\Gamma}}+\left(\left(A_{+}\right)^{-1}\right)_{\Gamma_{+} \backslash \breve{\Gamma}}\right) A \\
= & \left(\operatorname{diag}\left(e_{j_{1}}, \ldots, e_{j_{n}}\right)\right)\left(\left(A_{-}\right)_{\breve{\Gamma}}+\left(A_{-}\right)_{\Gamma_{-} \backslash \breve{\Gamma}}\right) .
\end{aligned}
$$

Since $j_{1}, \ldots, j_{n} \in \breve{\Gamma}$ and the Fourier spectrum of $A$ is contained in $\breve{\Gamma}$, (10) implies

$$
\left(A_{+}^{-1}\right)_{\breve{\Gamma}} A=\left(\operatorname{diag}\left(e_{j_{1}}, \ldots, e_{j_{n}}\right)\right)\left(A_{-}\right)_{\breve{\Gamma}} .
$$

Rewriting this equality in the form

$$
A=\left(\left(A_{+}^{-1}\right)_{\breve{\Gamma}}\right)^{-1}\left(\operatorname{diag}\left(e_{j_{1}}, \ldots, e_{j_{n}}\right)\right)\left(A_{-}\right)_{\breve{\Gamma}},
$$

we obtain a finitely generated factorization of $A$.

Theorem 7. Let $A$ be given as in Theorem 3 with $p=q$, and assume that (5) holds. If the matrix $c_{1}$ is invertible and the spectrum of $c_{1}^{-1} c_{2}$ does not intersect the unit circle, or if $c_{2}$ is invertible and the spectrum of $c_{2}^{-1} c_{1}$ does not intersect the unit circle, then $A$ admits a finitely generated factorization. Moreover, the factorization indices belong to the set $\{ \pm \sigma, \pm \mu\}$.

For the proof see [14]. In fact, the proof of Theorem 7 shows more detailed information about the factorization indices:

Theorem 8. Under the hypotheses of Theorem 7, assume that $c_{1}$ is invertible and the spectrum of $c_{1}^{-1} c_{2}$ does not intersect the unit circle, and let $r$ be the dimension of the spectral subspace of $c_{1}^{-1} c_{2}$ corresponding to the eigenvalues inside the unit circle. Then the factorization indices of $A$ are $\sigma$ ( $r$ times), $-\sigma$ ( $r$ times), $\mu(p-r$ times), and $-\mu$ ( $p-r$ times).

If $c_{2}$ is invertible and the spectrum of $c_{2}^{-1} c_{1}$ does not intersect the unit circle, then the factorization indices of $A$ are $\mu$ ( $r$ times), $-\mu$ ( $r$ times), $\sigma$ ( $p-r$ times), and $-\sigma$ ( $p-r$ times), where $r$ be the dimension of the spectral subspace of $c_{2}^{-1} c_{1}$ corresponding to the eigenvalues inside the unit circle.

Finally, we present a result concerning linearly ordered groups that will be used in the next section.

Proposition 9. Let $(\Gamma, \preceq)$ be a finitely generated additive ordered abelian group. Let $\Gamma_{0}$ stand for the additive subgroup of $\Gamma$ generated by all archimedean equivalence classes preceding the archimedean equivalence class $E$. Then there exists an additive subgroup $\Gamma_{1}$ of $\Gamma$ such that the direct sum decomposition

$$
\Gamma=\Gamma_{0} \dot{+} \Gamma_{1}
$$

holds and the coordinate projection $\Gamma \rightarrow \Gamma_{1}$ is $\preceq$-order preserving. 
Proof. With no loss of generality we assume that $\Gamma=\mathbb{Z}^{k}$ and that the order $\preceq$ on $\mathbb{Z}^{k}$ has been extended to a so-called term order on $\mathbb{R}^{k}$. That is, if $x \preceq y$ in $\mathbb{R}^{k}, z \in \mathbb{R}^{k}$ and $c \geq 0$, then $x+z \preceq y+z$ and $c x \preceq c y$. Such an extension is always possible but is often nonunique [3]. There now exists an orthonormal basis $\left\{e_{1}, \ldots, e_{k}\right\}$ of $\mathbb{R}^{k}$ and a decreasing sequence $\left\{H_{0}, H_{1}, \ldots, H_{k}\right\}$ of linear subspaces of $\mathbb{R}^{k}$ with $\operatorname{dim} H_{r}=$ $k-r(r=0,1, \ldots, k)$ such that $e_{r} \succ 0, e_{r} \in H_{r-1}$ and $e_{r} \perp H_{r}(r=1, \ldots, k)$ (cf. [5]). Here we note that the orthonormal basis is completely determined by the term order $\preceq$ on $\mathbb{R}^{k}$, with the one-to-one correspondence between term order (on $\mathbb{R}^{k}$ ) and orthonormal basis given by

$$
x=\left(x_{1}, \ldots, x_{k}\right) \succ(0, \ldots, 0) \Leftrightarrow\left\{\begin{array}{l}
x_{1}>0 \text { or } \\
x_{1}=0 \text { and } x_{2}>0, \text { or } \\
\vdots \\
x_{1}=\cdots=x_{k-1}=0 \text { and } x_{k}>0 .
\end{array}\right.
$$

Indeed, put $H_{0}=\mathbb{R}^{k}$ and let $H_{1}$ stand for the set of those points in $\mathbb{R}^{k}$ all of whose neighborhoods contain elements of both $\Gamma_{+}$and $\Gamma_{-}$. Then $H_{1}$ is a linear subspace of $\mathbb{R}^{k}$ of dimension $k-1$ [5]. We now let $e_{1}$ be the unique unit vector in $\mathbb{R}^{k}$ that is $\preceq$-positive and orthogonal to $H_{1}$ and restrict the term order to $H_{1}$. We now repeat the same construction in $H_{1}$ and find a linear subspace $H_{2}$ of $H_{1}$ of dimension $k-2$ and a unique $\preceq$-positive unit vector $e_{2}$ in $H_{1}$ orthogonal to $H_{2}$. After finitely many such constructions we arrive at the sequence of linear subspaces $\mathbb{R}^{k}=H_{0} \supset H_{1} \supset \cdots \supset H_{k-1} \supset H_{k}=\{0\}$ and the orthonormal basis $e_{1}, \ldots, e_{k}$ of $\mathbb{R}^{k}$ as indicated above.

Next, let $\tilde{H}_{r}$ be the smallest linear subspace of $\mathbb{R}^{k}$ spanned by $H_{r} \cap \mathbb{Z}^{k}$ $(r=0,1, \ldots, k)$. From this nonincreasing set of linear subspaces of $\mathbb{R}^{k}$ we select a maximal strictly decreasing set of nontrivial linear subspaces $\mathbb{R}^{k}=L_{0} \supset L_{1} \supset$ $\cdots \supset L_{\mu-1} \neq\{0\}$. Also let $\nu$ be the largest among the integers $s \in\{1, \ldots, k\}$ such that $L_{\mu-1}$ is spanned by $H_{s-1} \cap \mathbb{Z}^{k}$; then $H_{\nu} \cap \mathbb{Z}^{k}=\{0\}$. If $\mu=1$, we have $H_{1} \cap \mathbb{Z}^{k}=\{0\}$, so that the ordered group $\left(\mathbb{Z}^{k}, \preceq\right)$ is archimedean; in that case $i \mapsto \xi_{1}(i) \stackrel{\text { def }}{=}\left(i, e_{1}\right)$ (i.e., the signed distance from $i$ to $\left.H_{1}\right)$ is an order preserving group homomorphism from $\left(\mathbb{Z}^{k}, \preceq\right)$ into $\mathbb{R}$. On the other hand, if $\mu \geq 2$, we let (i) $\xi_{1}(i)$ stand for the signed distance from $i$ to $H_{1}$ and $p_{1}(i)$ for the orthogonal projection of $i$ onto $L_{1}$, (ii) $\xi_{r}(i)$ for the signed distance from $p_{r-1}(i)$ to $H_{q}$ for $q=\min \left\{s: L_{r}=\operatorname{span}\left(H_{s} \cap \mathbb{Z}^{k}\right)\right\}$ and $p_{r}(i)$ for the orthogonal projection of $p_{r-1}(i)$ onto $L_{r}(r=2, \ldots, \mu-1)$, and finally (iii) $\xi_{\mu}(i)$ as the signed distance from $p_{\mu-1}(i)$ to $H_{\nu}$. In this way

$$
i \stackrel{\varphi}{\mapsto}\left(\xi_{1}(i), \ldots, \xi_{\mu}(i)\right)
$$

is an order preserving group homomorphism from $\left(\mathbb{Z}^{k}, \preceq\right)$ into $\mathbb{R}^{\mu}$ with lexicographical order. It then appears that $\mu$ is the number of nontrivial (i.e., different from $\{0\}$ ) archimedean components. Moreover, in increasing order the archimedean components of $(\Gamma, \preceq)$ are now as follows:

$$
\boldsymbol{J}_{0}=\{0\}, \quad \boldsymbol{J}_{r}=\left[L_{\mu-r} \cap \Gamma_{+}\right] \backslash \cup_{s=0}^{r-1} \boldsymbol{J}_{s}(r=1, \ldots, \mu) .
$$


The additive subgroups of $\mathbb{Z}^{k}$ generated by the smallest archimedean components are as follows:

$$
\Gamma_{J_{0}}=\{0\}, \quad \Gamma_{J_{r}}=L_{\mu-r} \cap \Gamma(r=1, \ldots, \mu) .
$$

Let us now define the group homomorphisms $\pi_{r}$ on $\Gamma$ with image $\Gamma_{\boldsymbol{J}_{r}}$ and $q_{r}$ with kernel $\Gamma_{\boldsymbol{J}_{r}}$ by $\pi_{0}=0, q_{0}$ equal the identity, and

$$
\left\{\begin{array}{l}
\pi_{r} i=\varphi^{-1}\left(0, \ldots, 0, \xi_{\mu-r+1}(i), \ldots, \xi_{\mu}(i)\right), \\
q_{r} i=\varphi^{-1}\left(\xi_{1}(i), \ldots, \xi_{\mu-r}(i), 0, \ldots, 0\right) .
\end{array}\right.
$$

Then the fact that the linear order on $\varphi\left[\mathbb{Z}^{k}\right] \subset \mathbb{R}^{\mu}$ is lexicographical, implies that the additive group homomorphisms $q_{0}, q_{1}, \ldots, q_{\mu}$ are order preserving, but $\pi_{1}, \ldots, \pi_{\mu-1}$ are not. Putting $\Gamma_{\boldsymbol{J}_{r}}^{\prime}=q_{r}[\Gamma]$ we obtain the direct sum decomposition

$$
\Gamma=\Gamma_{\boldsymbol{J}_{r}} \dot{+} \Gamma_{\boldsymbol{J}_{r}}^{\prime}, \quad r=0,1, \ldots, \mu,
$$

which completes the proof.

\section{Proof of Theorem 3}

Using Theorem 6 we can assume without loss of generality that $\Gamma$ is finitely generated, and furthermore assume that $\Gamma=\mathbb{Z}^{k}$ for some positive integer $k$.

Consider the part "if". Applying the transformation

$$
c_{1} \mapsto S c_{1} T, \quad c_{2} \mapsto S c_{2} T,
$$

for suitable invertible matrices $S$ and $T$, we may assume that the pair $\left(c_{1}, c_{2}\right)$ is in the Kronecker normal form (see, e.g, [7]); in other words, $c_{1}$ and $c_{2}$ are direct sums of blocks of the following types:

(a) $c_{1}$ and $c_{2}$ are of size $k \times(k+1)$ of the form

$$
c_{1}=\left[\begin{array}{ll}
I_{k} & 0_{k \times 1}
\end{array}\right], \quad c_{2}=\left[\begin{array}{ll}
0_{k \times 1} & I_{k}
\end{array}\right] .
$$

(b) $c_{1}$ and $c_{2}$ are of size $(k+1) \times k$ of the form

$$
c_{1}=\left[\begin{array}{c}
I_{k} \\
0_{1 \times k}
\end{array}\right], \quad c_{2}=\left[\begin{array}{c}
0_{1 \times k} \\
I_{k}
\end{array}\right] .
$$

(c) $c_{1}$ is the $k \times k$ upper triangular nilpotent Jordan block, denoted by $V_{k}$, and $c_{2}=I_{k}$.

(d) $c_{1}=I_{k}$, and $c_{2}=V_{k}$.

(e) $c_{1}$ and $c_{2}$ are both invertible of the same size.

(f) $c_{1}$ and $c_{2}$ are both zero matrices of the same size.

Note that if $c_{1}$ (resp., $c_{2}$ ) is invertible, then condition (6) is equivalent to the condition that the spectrum of $c_{1}^{-1} c_{2}$ (resp., of $c_{1} c_{2}^{-1}$ ) does not intersect the unit circle. Thus, by Theorem 7 we are done in cases (c), (d), and (e), as well as in the trivial case (f). 
Consider the cases (a) and (b), where the condition (6) is obviously satisfied. We follow arguments similar to those presented in [13], and also in the proof of [14, Theorem 7].

Let $J_{k}$ be the $k \times k$ matrix with 1 's along the top-right to the left-bottom diagonal and zeros in all other positions. If $A(g)=\left[a_{i, j}(g)\right]_{i, j=1}^{n} \in(W(G))^{n \times n}$, then $A^{*}$ will denote the matrix function defined by $\left[\overline{a_{j, i}(g)}\right]_{i, j=1}^{n}$; clearly, $A^{*} \in(W(G))^{n \times n}$, and if $A \in\left(W(G)_{ \pm}\right)^{n \times n}$, then $A^{*} \in\left(W(G)_{\mp}\right)^{n \times n}$. The transformation

$$
A \mapsto\left[\begin{array}{cc}
0 & J_{k+1} \\
J_{k} & 0
\end{array}\right] A^{*}\left[\begin{array}{cc}
0 & J_{k} \\
J_{k+1} & 0
\end{array}\right]
$$

transforms the case (b) to the case (a). Thus, it will suffice to consider the case (a):

$$
A=\left[\begin{array}{ccc}
e_{\lambda} I_{k} & 0 & 0 \\
0 & e_{\lambda} & 0 \\
e_{\sigma} I_{k}-e_{\mu} V_{k} & h & e_{-\lambda} I_{k}
\end{array}\right], \quad \text { where } \quad h=\left[\begin{array}{c}
0_{(k-1) \times 1} \\
-e_{\mu}
\end{array}\right]
$$

Let

$$
\begin{gathered}
B_{+}=\left[\begin{array}{ccc}
I_{k}-e_{\mu-\sigma} V_{k} & b & -e_{\lambda-\sigma} I_{k} \\
0 & 1 & 0 \\
0 & 0 & \sum_{j=0}^{k-1} e_{j(\mu-\sigma)} V_{k}^{j}
\end{array}\right], \quad \text { where } b=\left[\begin{array}{c}
0_{(k-1) \times 1} \\
-e_{\mu-\sigma}
\end{array}\right], \\
B_{-}=\left[\begin{array}{ccc}
\sum_{j=0}^{k-1} e_{j(\mu-\sigma)-\lambda-\sigma} V_{k}^{j} & 0 & I_{k} \\
0 & 1 & 0 \\
-I_{k} & 0 & 0
\end{array}\right] .
\end{gathered}
$$

Clearly,

$$
B_{+} \in \mathcal{G}\left(\left(W(G)_{+}\right)^{(2 k+1) \times(2 k+1)}\right) \quad \text { and } \quad B_{-} \in \mathcal{G}\left(\left(W(G)_{-}\right)^{(2 k+1) \times(2 k+1)}\right)
$$

(the latter inclusion follows from (5) and from $\mu \succeq \sigma$ ). A direct computation shows that

where

$$
\Phi_{0}:=B_{+} A B_{-}=\left[\begin{array}{ccc}
e_{-\sigma} I_{k} & 0 & 0 \\
0 & e_{\lambda} & 0 \\
0 & h_{k} & e_{\sigma} I_{k}
\end{array}\right]
$$

$$
\left(h_{k}\right)^{T}=\left[\begin{array}{llll}
-e_{(k-1)(\mu-\sigma)+\mu} & \cdots & -e_{(\mu-\sigma)+\mu} & -e_{\mu}
\end{array}\right] .
$$

Define for $j=0,1, \ldots, k-1$ the auxiliary matrices

$$
\begin{gathered}
R_{+, k-j}=\left[\begin{array}{ccc}
1 & 0 & e_{\lambda-\mu-j(\mu-\sigma)} \\
0 & I_{k-j-1} & h_{k-j-1} e_{-\sigma} \\
0 & 0 & 1
\end{array}\right], \\
R_{-, k-j}=\left[\begin{array}{ccc}
e_{\sigma-\mu} & 0 & -1 \\
0 & I_{k-j-1} & 0 \\
1 & 0 & 0
\end{array}\right], \quad R_{k-j}=\left[\begin{array}{cc}
e_{\lambda-j(\mu-\sigma)} & 0 \\
h_{k-j} & e_{\sigma} I_{k-j}
\end{array}\right] .
\end{gathered}
$$

Clearly, $R_{-, k-j} \in \mathcal{G}\left(\left(W(G)_{-}\right)^{(k-j+1) \times(k-j+1)}\right)$, and in view of (5),

$$
R_{+, k-j} \in \mathcal{G}\left(\left(W(G)_{+}\right)^{(k-j+1) \times(k-j+1)}\right) .
$$


We also have the recurrence relations

$$
R_{+, k-j} R_{k-j} R_{-, k-j}=\left[\begin{array}{cc}
R_{k-j-1} & 0 \\
0 & e_{\mu}
\end{array}\right], \quad R_{0}=e_{\lambda-k(\mu-\sigma)},
$$

for $j=0, \ldots, k-1$. Note that $\Phi_{0}=\operatorname{diag}\left(e_{-\sigma} I_{k}, R_{k}\right)$. Applying consecutively (16) for $j=0, \ldots, k-1$, we obtain a factorization $A=A_{+} \Lambda A_{-}$with $\Lambda=$ $\operatorname{diag}\left(e_{-\sigma} I_{k}, e_{\lambda-k(\mu-\sigma)}, e_{\mu} I_{k}\right)$. This completes the proof of the "if" part of the theorem.

For the part "only if", we make use of the archimedean structure on $\Gamma=$ $\left(\mathbb{Z}^{k}, \preceq\right)$ (see the previous section). Let $\Gamma_{0}$ be the subgroup of $\Gamma$ generated by all archimedean classes of $\Gamma$ that are $\prec \lambda$. Condition (5) guarantees that $0 \neq \mu-\sigma \in \Gamma_{0}$ and hence that $\Gamma_{0} \neq\{0\}$. Since

$$
\alpha \in \Gamma, \quad n \alpha \in \Gamma_{0} \quad \text { for some } n \in \mathbb{N} \quad \Longrightarrow \quad \alpha \in \Gamma_{0},
$$

it follows that

$$
\Gamma=\Gamma_{0} \dot{+} \Gamma_{1},
$$

a direct sum, for some subgroup $\Gamma_{1}$ of $\Gamma=\mathbb{Z}^{k}$, where the coordinate projection onto $\Gamma_{1}$ along $\Gamma_{0}$ is order preserving (Proposition 9). Also, by [11, Theorem 23.18], we may assume

$$
G=G_{0} \times G_{1}
$$

where $G_{j}$ is the character group of $\Gamma_{j}, j=0,1$. We write

$$
\lambda=\lambda_{0}+\lambda_{1}, \quad \mu=\mu_{0}+\mu_{1}, \quad \sigma=\sigma_{0}+\sigma_{1},
$$

in accordance with (17). By construction of $\Gamma_{0}$, we have $\lambda_{1} \succ 0$, and by (5) $\mu, \sigma \in \Gamma_{0}$, and so $\mu_{1}=\sigma_{1}=0$.

Assume that $A$ has a factorization

$$
A(g)=A_{+}(g)\left(\operatorname{diag}\left(e_{j_{1}}(g), \ldots, e_{j_{n}}(g)\right)\right) A_{-}(g), \quad g \in G .
$$

In accordance with (17) and (18) write

$$
\begin{gathered}
j_{k}=j_{k, 0}+j_{k, 1}, \quad j_{k, 0} \in \Gamma_{0}, \quad j_{k, 1} \in \Gamma_{1}, \quad k=1, \ldots, n, \\
g=g_{0} g_{1}, \quad g_{0} \in G_{0}, \quad g_{1} \in G_{1},
\end{gathered}
$$

and consider the equation (19) in which $g_{0}$ is kept fixed, whereas $g_{1}$ is kept variable. To emphasize this interpretation, we write (19) in the form

$$
\begin{aligned}
A_{g_{0}}\left(g_{1}\right)= & A_{+, g_{0}}\left(g_{1}\right)\left(\operatorname{diag}\left(e_{j_{1,0}}\left(g_{0}\right), \ldots, e_{j_{n, 0}}\left(g_{0}\right)\right)\right) \\
& \cdot\left(\operatorname{diag}\left(e_{j_{1,1}}\left(g_{1}\right), \ldots, e_{j_{n, 1}}\left(g_{1}\right)\right)\right) A_{-, g_{0}}\left(g_{1}\right) .
\end{aligned}
$$

We consider $\Gamma_{1}$ with the linear order induced by $(\Gamma, \preceq)$. Since the property that $\alpha=\alpha_{0}+\alpha_{1} \in \Gamma_{ \pm}$, where $\alpha_{j} \in \Gamma_{j}, j=0,1$, implies that $\alpha_{1} \in\left(\Gamma_{1}\right)_{ \pm}$, we obtain

$$
A_{ \pm, g_{0}} \in \mathcal{G}\left(\left(W\left(G_{1}\right)_{ \pm}\right)^{n \times n}\right)
$$

for every $g_{0} \in \Gamma_{0}$. Thus, $(20)$ is in fact a factorization of $A_{g_{0}}\left(g_{1}\right)$ whose factorization indices are $j_{1,1}, \ldots, j_{n, 1}$, and moreover we have the following property:

(※) the factorization indices of $A_{g_{0}}\left(g_{1}\right)$ are independent of $g_{0} \in G_{0}$. 
Arguing by contradiction, we assume that

$$
\begin{aligned}
\operatorname{rank}\left(\lambda_{1} c_{1}-\lambda_{2} c_{2}\right)< & \max \left\{\operatorname{rank}\left(z_{1} c_{1}-z_{2} c_{2}\right): z_{1}, z_{2} \in \mathbb{C}\right\} \\
& \text { for some } \lambda_{1}, \lambda_{2} \in \mathbb{C} \text { satisfying }\left|\lambda_{1}\right|=\left|\lambda_{2}\right|=1
\end{aligned}
$$

A contradiction will be obtained with Property $(\aleph)$. We can assume, using the Kronecker normal from (see, e.g., [7]), that $c_{1}$ and $c_{2}$ have the form

$$
c_{1}=\operatorname{diag}\left(c_{1,1}, \ldots, c_{1, s}\right), \quad c_{2}=\operatorname{diag}\left(c_{2,1}, \ldots, c_{2, s}\right),
$$

where each pair of blocks $\left(c_{1, w}, c_{2, w}\right)$ has one of the forms (a) - (f). After a permutation transformation, we obtain (keeping the same notation for the transformed $\left.A_{g_{0}}\left(g_{1}\right)\right)$ :

$$
A_{g_{0}}\left(g_{1}\right)=\operatorname{diag}\left(A_{g_{0}, 1}\left(g_{1}\right), \ldots, A_{g_{0}, s}\left(g_{1}\right)\right),
$$

where

$$
A_{g_{0}, w}\left(g_{1}\right)=\left[\begin{array}{cc}
e_{\lambda_{1}}\left(g_{1}\right) I_{p_{w}} & 0 \\
c_{1, w} e_{\beta}\left(g_{0}\right)-c_{2, w} e_{\kappa}\left(g_{0}\right) & e_{-\lambda_{1}}\left(g_{1}\right) I_{q_{w}}
\end{array}\right] Q, \quad w=1, \ldots, s,
$$

with $\beta, \kappa \in \Gamma_{0}$ independent of $w$, and $Q$ is a diagonal matrix (also independent of $w$ ) with terms of the form $e_{\alpha}\left(g_{0}\right), \alpha \in \Gamma_{0}$ on the main diagonal. Note that $\beta \neq \kappa$ (otherwise we would have $\mu=\sigma$, which is excluded by the hypotheses of the theorem). The "if" part of the theorem shows that $A_{g_{0}, w}\left(g_{1}\right)$ is factorable with indices independent of $g_{0}$ if the pair $\left(c_{1, w}, c_{2, w}\right)$ has one of the forms (a), (b), (c), (d), and (f).

Suppose that the pair $\left(c_{1, w}, c_{2, w}\right)$ is of the form (e). Then we may further assume that $c_{1, w}=I$ and $c_{2, w}$ is in the Jordan form:

$$
c_{2, w}=J_{\tau_{1}}\left(\rho_{1}\right) \oplus \cdots \oplus J_{\tau_{u}}\left(\rho_{u}\right),
$$

where $J_{\tau_{j}}\left(\rho_{j}\right)$ is the upper triangular $\tau_{j} \times \tau_{j}$ Jordan block with the eigenvalue $\rho_{j}$ (for notational simplicity, we suppress the dependence of $\rho_{j}, \tau_{j}$, and $u$ on $w$ in the notation used). Accordingly, after a permutation transformation we have $A_{g_{0}, w}\left(g_{1}\right) Q^{-1}$ in the following form:

$$
\tilde{A}_{g_{0}, w}\left(g_{1}\right)=\operatorname{diag}\left(\tilde{A}_{g_{0}, w, 1}\left(g_{1}\right), \ldots, \tilde{A}_{g_{0}, w, u}\left(g_{1}\right)\right),
$$

where

$$
\tilde{A}_{g_{0}, w, j}\left(g_{1}\right)=\left[\begin{array}{cc}
e_{\lambda_{1}}\left(g_{1}\right) I_{\tau_{j}} & 0 \\
e_{\beta}\left(g_{0}\right) I_{\tau_{j}}-e_{\kappa}\left(g_{0}\right) J_{\tau_{j}}\left(\rho_{j}\right) & e_{-\lambda_{1}}\left(g_{1}\right) I_{\tau_{j}}
\end{array}\right], j=1, \ldots, u .
$$

If $\left|\rho_{j}\right| \neq 1$, then by the "if" part of the theorem, the factorization indices of $\tilde{A}_{g_{0}, w, j}\left(g_{1}\right)$ are independent of $g_{0}$ (this can be also checked directly). Assume $\left|\rho_{j}\right|=$ 1 ; then the factorization indices of $\tilde{A}_{g_{0}, w, j}\left(g_{1}\right)$ equal zero if

$$
e_{\beta}\left(g_{0}\right)-\rho_{j} e_{\kappa}\left(g_{0}\right) \neq 0 \text {. }
$$

Indeed,

$$
\left[\begin{array}{cc}
e_{\lambda_{1}} I & 0 \\
S & e_{-\lambda_{1}} I
\end{array}\right]=\left[\begin{array}{cc}
I & e_{\lambda_{1}} S^{-1} \\
0 & I
\end{array}\right]\left[\begin{array}{cc}
0 & -S^{-1} \\
S & e_{-\lambda_{1}} I
\end{array}\right]
$$


where

If $\left|\rho_{j}\right|=1$ and

$$
S=e_{\beta}\left(g_{0}\right) I_{\tau_{j}}-e_{\kappa}\left(g_{0}\right) J_{\tau_{j}}\left(\rho_{j}\right)
$$

$$
e_{\beta}\left(g_{0}\right)-\rho_{j} e_{\kappa}\left(g_{0}\right)=0,
$$

then the factorization indices of $\tilde{A}_{g_{0}, w, j}\left(g_{1}\right)$ are zeros $\left(2\left(\tau_{j}-1\right)\right.$ times $)$ and $\pm \lambda_{1}$. It follows that for the values of $g_{0}$ such that

$$
e_{\beta}\left(g_{0}\right)-\rho e_{\kappa}\left(g_{0}\right) \neq 0
$$

for any eigenvalue $\rho$ of $c_{2, w}$ the factorization indices of $A_{g_{0}, w}\left(g_{1}\right)$ are all zeros, whereas in case the equality

$$
e_{\beta}\left(g_{0}\right)-\rho e_{\kappa}\left(g_{0}\right)=0
$$

holds for some eigenvalue $\rho$ of $c_{2, w}$ not all factorization indices of $A_{g_{0}, w}\left(t_{1}\right)$ are zeros. Since $\kappa \neq \beta$, the range of the function

$$
e_{\beta-\kappa}\left(g_{0}\right)=e_{\beta}\left(g_{0}\right)\left(e_{\kappa}\left(g_{0}\right)\right)^{-1}
$$

coincides with the unit circle (since $G$ is connected and the characters are continuous), and therefore by hypothesis (21) there do exist eigenvalues $\rho$ of $c_{2, w}$ for which (25) holds. We obtain a contradiction with Property $\aleph$.

This completes the proof of Theorem 3 .

\section{Invertibility vs factorizability}

The following conjecture was stated in [14].

Conjecture 10. Every function $A \in \mathcal{G}\left(W(G)^{n \times n}\right)$ admits a factorization if and only if $\Gamma$ (as an abstract group without regard to $\succeq$ ) is isomorphic to a subgroup of the additive group of rational numbers $\mathbb{Q}$.

Regarding this conjecture we quote a result from [14]:

Theorem 11. If $\Gamma$ is not isomorphic to a subgroup of $\mathbb{Q}$, then there exists a $2 \times 2$ matrix function of the form

$$
A(g)=\left[\begin{array}{cc}
e_{\lambda}(g) & 0 \\
c_{1} e_{\alpha_{1}}(g)+c_{2}+c_{3} e_{\alpha_{3}}(g) & e_{-\lambda}(g)
\end{array}\right], \quad g \in G,
$$

where $\lambda, \alpha_{1}, \alpha_{2}, \alpha_{3} \in \Gamma$, and $c_{1}, c_{2}, c_{3} \in \mathbb{C}$, which does not admit a factorization with the factors $A_{ \pm}$and their inverses $A_{ \pm}^{-1}$ having finite Fourier spectrum.

We improve on Theorem 11:

Theorem 12. If $\Gamma$ is not isomorphic (as an abstract group) to a subgroup of $\mathbb{Q}$, then there exists a $2 \times 2$ matrix function of the form (26) which is not factorable.

Proof. Consider two cases: (1) $\Gamma$ is archimedean. Then $(\Gamma, \succeq)$ is isomorphic to a subgroup of the additive group of real numbers (Hölder's theorem, see, e.g., [6]) and since $\Gamma$ is not isomorphic to a subgroup of $\mathbb{Q}$, there exist non-commensurable 
elements $x, y \in \Gamma \backslash\{0\}$. Using $x$ and $y$, a known construction (see [12], also [2, Section 8.5]) may be used to produce a $2 \times 2$ matrix function of the required form.

(2) $\Gamma$ is not archimedean. Then there exist $\sigma=0 \prec \mu \prec \lambda \in \Gamma$ such that (5) holds. Theorem 3 now implies that the function

is not factorable.

$$
\left[\begin{array}{cc}
e_{\lambda}(g) & 0 \\
1-e_{\mu}(g) & e_{-\lambda}(g)
\end{array}\right], \quad g \in G
$$

Theorem 12 and its proof show that if $\Gamma$ is not isomorphic to a subgroup of $\mathbb{Q}$, then there exists a $2 \times 2$ matrix function of the form

$$
A(g)=\sum_{j=1}^{k} c_{j} e_{\alpha_{j}}(g), \quad \operatorname{det} A(g) \equiv 1,
$$

which is not factorable, with $k=5$ if $\Gamma$ is archimedean, and $k=4$ if $\Gamma$ is not archimedean. On the other hand, for every linearly ordered group $\Gamma$, every $n \times n$ matrix function of the form

$$
A(g)=c_{1} e_{\alpha_{1}}(g)+c_{2} e_{\alpha_{2}}(g)
$$

with $\operatorname{det} A(g) \neq 0, g \in G$, is factorable. Indeed, this follows easily from the Kronecker form of the pair of matrices $\left(c_{1}, c_{2}\right)$. This leaves the following problem open:

Problem 13. Assume that $\Gamma$ is not isomorphic to a subgroup of $\mathbb{Q}$.

(a) If the subgroup generated by $\alpha_{1}, \alpha_{2}, \alpha_{3} \in \Gamma$ is not archimedean, prove or disprove that every $n \times n$ matrix function of the form

$$
A(g)=c_{1} e_{\alpha_{1}}(g)+c_{2} e_{\alpha_{2}}(g)+c_{3} e_{\alpha_{3}}(g)
$$

with $\operatorname{det} A(g) \neq 0, g \in G$, is factorable.

(b) If $\Gamma$ is archimedean, prove or disprove that every $n \times n$ matrix function of the form $A(g)=\sum_{j=1}^{k} c_{j} e_{\alpha_{j}}(g)$ with $\operatorname{det} A(g) \neq 0, g \in G$, and with $k=3$ or $k=4$, is factorable.

\section{References}

[1] G.R. Allan, One-sided inverses in Banach algebras of holomorphic vector-valued functions, J. London Math. Soc. 42, 463-470 (1967).

[2] A. Böttcher, Yu.I. Karlovich, and I.M. Spitkovsky, Convolution Operators and Factorization of Almost Periodic Matrix Functions, Birkhäuser OT 131, Basel and Boston, 2002.

[3] L. Cerlienco and M. Mureddu, Rappresentazione matriciale degli ordini l.c. su $\mathbb{R}^{n}$ e su $\mathbb{N}^{n}$, Rend. Sem. Fac. Sc. Univ. Cagliari 66, 49-68 (1996).

[4] K.F. Clancey and I. Gohberg, Factorization of Matrix Functions and Singular Integral Operators, Birkhäuser OT 3, Basel and Boston, 1981.

[5] J. Erdős, On the structure of ordered real vector spaces, Publ. Math. Debrecen 4, 334-343 (1956).

[6] L. Fuchs, Partially Ordered Algebraic Systems, Pergamon Press, Oxford, 1963. 
[7] F.R. Gantmacher, Applications of the Theory of Matrices, Interscience Publishers, New York, 1959. (Translation from Russian.)

[8] I.C. Gohberg and Yu. Leiterer, Factorization of operator functions with respect to a contour. II. Canonical factorization of operator functions close to the identity, Math. Nachr. 54, 41-74 (1972). (Russian)

[9] I.C. Gohberg and I.A. Feldman, Convolution Equations and Projection Methods for their Solution, Transl. Math. Monographs 41, Amer. Math. Soc., Providence, R. I., 1974.

[10] I.C. Gohberg and M.G. Krein, Systems of integral equations on a half line with kernels depending on the difference of arguments, Amer. Math. Soc. Transl. (2)14, 217-287 (1960).

[11] E. Hewitt and K.A. Ross, Abstract Harmonic Analysis I, 2nd edition, SpringerVerlag, Berlin, Heidelberg, New York, 1979.

[12] Yu.I. Karlovich and I.M. Spitkovsky, On the Noether property for certain singular integral operators with matrix coefficients of the class SAP and the systems of convolution equations on a finite interval connected with them, Soviet Math. Doklady 27, 358-363 (1983).

[13] Yu.I. Karlovich and I.M. Spitkovsky, Factorization of almost periodic matrix functions and (semi)-Fredholmness of some convolution type equations, No. 4421-85 dep., VINITI, Moscow, 1985. (Russian).

[14] C.V.M. van der Mee, L. Rodman, I.M. Spitkovsky, and H. J. Woerdeman, Factorization of block triangular matrix functions in Wiener algebras on ordered abelian groups. In: J.A. Ball, J.W. Helton, M. Klaus, and L. Rodman (eds.), Current Trends in Operator Theory and its Applications, Birkhäuser OT 149, Basel and Boston, 2004, pp. 441-465.

[15] L. Rodman, I.M. Spitkovsky, and H.J. Woerdeman, Carathéodory-Toeplitz and Nehari problems for matrix-valued almost periodic functions, Trans. Amer. Math. Soc. 350, 2185-2227 (1998).

[16] L. Rodman, I.M. Spitkovsky, and H.J. Woerdeman, Noncanonical factorizations of almost periodic multivariable matrix functions, Operator Theory: Advances and Applications 142, 311-344 (2003).

[17] W. Rudin, Fourier Analysis on Groups, John Wiley, New York, 1962.

Cornelis V.M. van der Mee

Dipartimento di Matematica e Informatica

Università di Cagliari

Viale Merello 92

I-09123 Cagliari, Italy

e-mail: cornelis@bugs . unica.it

Leiba Rodman and Ilya M. Spitkovsky

Department of Mathematics

The College of William and Mary

Williamsburg, VA 23187-8795, USA

e-mail: lxrodm@math.wm.edu

e-mail: ilya@math.wm.edu 